Draft VERSION OCTOBER 2, 2018

Preprint typeset using $\mathrm{LATE}_{\mathrm{E}} \mathrm{X}$ style emulateapj v. 5/2/11

\title{
SOLAR NEUTRINO PHYSICS OSCILLATIONS: SENSITIVITY TO THE ELECTRONIC DENSITY IN THE SUN'S CORE
}

\author{
Ilídio Lopes ${ }^{1,2,4}$ AND Sylvaine TurCK-Chièze ${ }^{3,5}$ \\ Draft version October 2, 2018
}

\begin{abstract}
Solar neutrinos coming from different nuclear reactions are now detected with a high statistics. Consequently, an accurate spectroscopic analysis of the neutrino fluxes arriving on the Earth's detectors become available, in the context of neutrino oscillations. In this work, we explore the possibility of using this information to infer the radial profile of the electronic density in the solar core. So, we discuss the constraints on the Sun's density and chemical composition that can be determined from solar neutrino observations. This approach constitutes an independent and alternative diagnostic to the helioseismic investigations already done. The direct inversion method, that we propose to get the radial solar electronic density profile, is almost independent of the solar model.
\end{abstract}

Subject headings: Neutrinos - Sun:evolution -Sun:interior - Stars: evolution -Stars:interiors

\section{INTRODUCTION}

Neutrinos, once produced in the core of the Sun, reach the Earth in less than 8 minutes. On their interplanetary journey, solar neutrinos very rarely interact with other particles. Luckily for physicists, neutrinos with a scattering cross-section varying from some $10^{-46} \mathrm{~cm}^{2}$ to some $10^{-42} \mathrm{~cm}^{2}$ (Bahcall 1989), occasionally interact with the baryons present in the solar neutrino detectors. Therefore, the neutrinos produced in the nuclear reactions of proton-proton (PP) chains and Carbon-Nitrogen-Oxygen (CNO) cycle are natural probe-particles of the constitutive plasma of the Sun's core.

The current theory of neutrino physics, following in the footsteps of the fundamental theory of elementary particles, has shown that neutrinos appear in three (at least) flavours (Hernandez 2010): electron-neutrino $\left(\nu_{e}\right)$, muon-neutrino $\left(\nu_{\mu}\right)$ and tau-neutrino $\left(\nu_{\tau}\right)$. The theory states that by vacuum flavour oscillations, when moving in vacuum, neutrinos have the ability to switch cyclically between different flavours. This mechanism of neutrino oscillations was proposed by Pontecorvo around 1940. In the late seventies, Lincoln Wolfenstein and colleagues suggested another mechanism for neutrinos to change flavour, as neutrinos move through a high density medium (Wolfenstein 1978): in an environment of high density material, the effective mass of the propagating neutrinos is different from the mass of neutrinos that propagates in a vacuum. Since the oscillations between different flavours of neutrinos depend on their mass, the oscillations of neutrinos in dense media are different from neutrino oscillations in vacuum. This process is now known as Mikheyev-Smirnov-Wolfenstein (MSW) or "matter oscillations". These two mechanisms of flavour

\footnotetext{
${ }^{1}$ Centro Multidisciplinar de Astrofísica, Instituto Superior Técnico, Universidade Tecnica de Lisboa, Av. Rovisco Pais, 1049-001 Lisboa, Portugal

${ }^{2}$ Departamento de Física,Escola de Ciencia e Tecnologia, Universidade de Évora, Colégio Luis António Verney, 7002-554 Évora - Portugal

3 CEA/IRFU/Service d'Astrophysique, CE Saclay, 91191 Gif sur Yvette, France

${ }^{4}$ E-mail:ilidio.lopes@ist.utl.pt;ilopes@uevora.pt

${ }^{5}$ E-mail:sylvaine.turck-chieze@cea.fr
}

oscillations are central to the modern theory of neutrinos. Through these mechanisms the electron-neutrinos produced in the Sun's core have a non-zero probability of being detected as a muon-neutrino or tau-neutrino when they reach the Earth. In particular a small fraction of electron-neutrinos changes flavour in the Sun's interior when they propagate through the high density plasma medium of the Sun's core.

In recent years, significant progress has been achieved in the understanding of the mechanism responsible for the neutrino oscillations (Eguchi et al. 2003; Fukuda et al. 2001; Ahmad et al. 2001). The current theory of neutrino physics successfully explains the neutrino observations. Furthermore, the theory has been used successfully to determine the fundamental properties of neutrinos (Schwetz et al. 2008).

If, during several decades, the solar neutrino fluxes arriving on Earth have been difficult to interpret, this is no longer the case as the flavour oscillations at different energies are well understood. In particular, the production of these neutrinos has been confirmed by helioseismic data. One may consider as a success that these two disciplines (solar neutrinos and helioseismology) agree within the error bars (see Turck-Chièze et al. 2010; Turck-Chièze \& Couvidat 2011, and references therein). Such an agreement, will now allows us to progress a step further. We believe that solar neutrinos will very likely become a powerful tool to probe the core of the Sun because the production of neutrinos is very sensitive to the local properties of the solar plasma.

In this paper, we focus on the sensitivity of solar neutrinos to the plasma in the solar core, and explore the possibilities of probing the physical properties of such plasma by using the solar neutrino flux measurements. This point has been proposed by John Bahcall and Raymond Davis about 50 years ago (Davis 1964). In principle, the structure of the solar core can be studied by means of neutrino spectroscopy in two fundamental ways: (i) to diagnose the temperature profile in the Sun's core, by measuring the total number of electronneutrinos produced in each nuclear reaction of $\mathrm{PP}$ chains and $\mathrm{CNO}$ cycle, in particular the boron flux that is the 


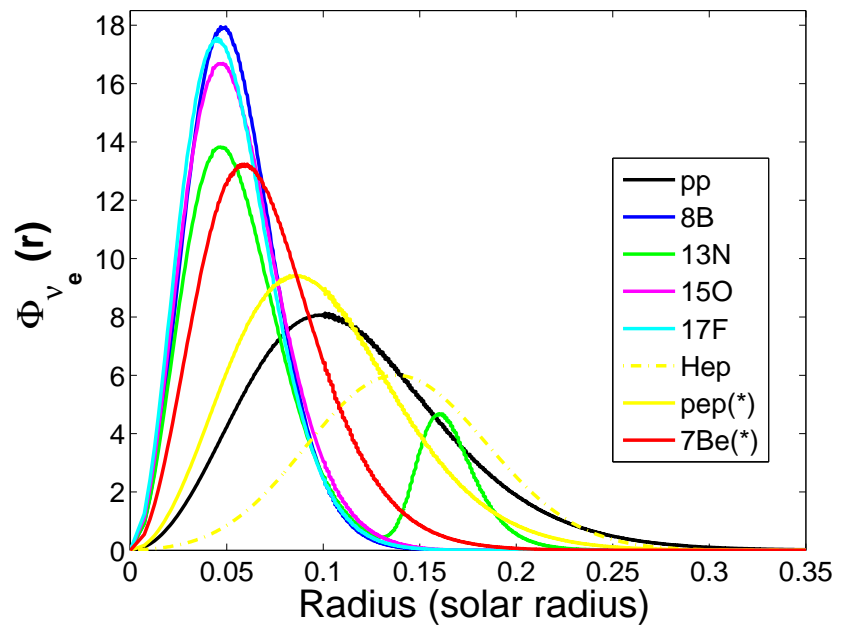

FIG. 1.- The electron-neutrino fluxes produced in the various nuclear reactions of the PP chains and CNO cycle. The center of the different neutrino sources $\Phi_{j}(r),\langle r\rangle_{j}$ (with $j=$ pep, $p p,{ }^{8} \mathrm{~B},{ }^{7} \mathrm{Be},{ }^{13} \mathrm{~N},{ }^{15} \mathrm{O},{ }^{17} \mathrm{~F}$ ) are the following ones (in unity of $\left.R_{\odot}\right): 0.099$ (pep), $0.117(p p), 0.125(\mathrm{Hep}), 0.054\left({ }^{8} \mathrm{~B}\right), 0.070\left({ }^{7} \mathrm{Be}\right)$, $0.074\left({ }^{13} N\right), 0.055\left({ }^{15} O\right), 0.053\left({ }^{17} F\right)$. These neutrino fluxes were calculated for the standard solar model, using the most updated microscopic physics data. This solar model is in agreement with the most current helioseismology diagnostic and other solar standard models published in the literature (see text). For each neutrino type $j, \Phi_{j}(r) \equiv\left(1 / F_{j}\right) d f_{j}(r) / d r$ is drawn as a function of the fractional radius $r$ for which $f_{j}$ is the flux in $\mathrm{s}^{-1}$ and $F_{j}$ is the total flux for this neutrino type.

most sensitive to the temperature (Turck-Chièze et al. 2012; Turck-Chièze \& Lopes 2012) and (ii) to measure the radial electronic density of matter of the Sun by determining the amount of electron-neutrinos that are converted into another flavour. This article is a first attempt to study this second physical information through neutrino probes.

The neutrino flavour oscillations by the MSW mechanism are particularly significant in the solar interior, where there is a wide radial variation of the plasma density. The standard solar model (Turck-Chièze \& Lopes 1993) partly validated by helioseismology, predicts that the density inside the Sun varies from about $150 \mathrm{~g} \mathrm{~cm}^{-3}$ in the centre of the star, to $1 \mathrm{~g} \mathrm{~cm}^{-3}$ at half of the solar radius.

What makes this diagnostic particularly powerful is the possibility of getting a direct measurement of the plasma electronic density almost independently of the solar model. The strong dependence of neutrino oscillations with the local electronic density of matter opens the possibility of inferring the matter density and chemical composition in the nuclear region. Since the neutrino oscillations in matter depend strongly on the matter density and weakly on the chemical composition, this is at first, a diagnosis of the radial density profile of matter in the solar core. The composition diagnostic can come from precise CNO neutrino detections. Therefore, we can anticipate that with the expected increased accuracy of the solar neutrino detections in the future, it will be possible to extend this analysis to the solar core chemical composition determination.

\section{CURRENT MODEL OF NEUTRINO PHYSICS OSCILLATIONS}

The theory of neutrino oscillations in vacuum and matter (Hernandez 2010; Schwetz et al. 2008) has successfully addressed the problem of the solar neutrino deficit - the discrepancy between the neutrino flux detected and the theoretical predictions of the solar standard model (Turck-Chièze \& Lopes 1993; Fogli et al. 2009; Gonzalez-Garcia \& Maltoni 2008). The theory provides a theoretical solution in full agreement with all solar neutrino experiments (Bellini et al. 2011b, 2010; Aharmim et al. 2010) as well as with the data obtained by the KamLAND reactor experiment (Eguchi et al. 2003). Presently, the theoretical model for neutrino flavour oscillations is defined by means of six parameters: the difference of the squared characteristic masses $\left(\Delta m_{12}^{2}, \Delta m_{31}^{2}\right)$, the mixing angles $\left(\theta_{12}, \theta_{23}, \theta_{31}\right)$ and the CP-violation phase. The mass square differences and mixing angles are known with reasonable accuracy (Fogli et al. 2009): $\Delta m_{31}^{2}$ is obtained from the experiments of atmospheric neutrinos and $\Delta m_{12}^{2}$ is obtained from solar neutrino experiments.

The mixing angles are not uniformly well defined: $\theta_{12}$ is obtained from solar neutrino experiments with an excellent precision; $\theta_{23}$ is obtained from the atmospheric neutrino experiments, this is the mixing angle of the highest value; $\theta_{13}$ has been first estimated from the Chooz reactor (Apollonio \& Baldini 1999), its value is very small and was still very uncertain (Fogli et al. 2009). Nowadays with Daya Bay and Reno, the situation is largely improved (Fogli et al. 2012), as shown in the next paragraph. However, present experiments cannot fix the value of the CP-violation phase (Hernandez 2010).

An overall fit to the data obtained from the different neutrino experiments: solar neutrino detectors, accelerators, atmospheric neutrino detectors and nuclear reactor experiments suggests that the parameters of neutrino oscillations are the following ones (Gonzalez-Garcia \& Maltoni 2008): $\Delta m_{31}^{2} \sim 2.46 \pm$ $0.1210^{-3} \mathrm{eV}^{2}, \Delta m_{12}^{2} \sim 7.59 \pm 0.2010^{-5} \mathrm{eV}^{2}, \theta_{12}=$ $(34.4 \pm 1)^{o}, \theta_{23}=\left(42.8_{-2.9}^{+4.7}\right)^{o}$ and $\theta_{13}=\left(5.6_{-2.7}^{+3.0}\right)^{o}$. The recent progress leads to $\theta_{13}=\left(8.6_{-0.46}^{+0.44}\right)^{\circ}$ and $\Delta m_{32}^{2} \sim$ $-2.43_{-0.06}^{+0.42} 10^{-3} \mathrm{eV}^{2}$ (Gonzalez-Garcia et al. 2012).

In the limiting case where the value of the mass differences, $\Delta m_{12}^{2}$ or $\Delta m_{31}^{2}$ is large, or one of the angles of mixing $\left(\theta_{12}, \theta_{23}, \theta_{31}\right)$ is small, the theory of three neutrino flavour oscillations reverts to an effective theory of two neutrino flavour oscillations (Hernandez 2010). Balantekin and Yuksel have shown that the survival probability of solar neutrinos calculated in a model with two neutrino flavour oscillations or three neutrino flavour oscillations have very close values Balantekin \& Yuksel 2003).

In the present work, for reasons of convenience and simplicity, we will restrict our study to the theory of two neutrino flavour oscillations. The generalization of the results to a theory of three neutrino flavour oscillations is obvious (Lisi \& Montanino 1997; Gando et al. 2011). The survival probability of electron-neutrino function $P_{\nu_{e}}\left(\equiv P\left(\nu_{e} \rightarrow \nu_{e}\right)\right)$ in a two flavour neutrino theory is given by

$$
P_{\nu_{e}}(E, r)=\frac{1}{2}+F(\gamma) \cos \left(2 \theta_{v}\right) \cos \left(2 \theta_{m}\right)
$$

where $\theta_{v}$ is the angle of oscillation in vacuum and $\theta_{m}$ 
is the angle oscillation in matter. The function $F(\gamma)$ is a first-order correction to the adiabatic approximation of the propagation of neutrinos in matter, where $\gamma$ is the adiabatic parameter which depends of the local properties of the plasma (Landau \& Rosenkewitsch 1932). The adiabaticity occurs when the electronic density of the propagating medium is a slow varying function over the neutrino travel path (or equivalently the solar radius). This approximation is valid in the solar interior, in the case where $\gamma \gg 1$, a condition that is verified in much of the solar core and the radiative region (Landau \& Rosenkewitsch 1932; Zener 1932). As $F(\gamma)=0.5-P_{12}$ where $P_{12}$ corresponds to the transition between two distinct neutrino flavours, at first order it is reasonable to consider that $P_{12}=0$. The numerical value of $F(\gamma)$ is approximately equal to 0.5 (Bilenky 2010). The angle $\theta_{v}$ depends on the distance from the Sun to the Earth. In this model we assume $\theta_{v}$ to be equal to $\theta_{12}$. The phase $\theta_{m}$ is the most important term in this analysis, since it depends on the properties of the plasma in the Sun's core, namely, the local electron density. The neutrino mixing angle $\theta_{m}$ is given by

$$
\sin 2 \theta_{m}=\frac{\sin 2 \theta_{v}}{\sqrt{\left(V / \Delta m^{2}-\cos \left(2 \theta_{v}\right)\right)^{2}+\sin ^{2}\left(2 \theta_{v}\right)}}
$$

where $\Delta m^{2}$ is the difference of the squared masses we consider to be equal to $\Delta m_{12}^{2}$. The function of the solar plasma $V$ is given by

$$
V(E, r)=2 \sqrt{2} G_{f} n_{e}(r) E
$$

where $G_{f}$ is the Fermi constant, $n_{e}(r)$ is the electron density of plasma and $E$ the energy of the neutrino. The electron density $n_{e}(r)=N_{o} \rho(r) / \mu_{e}(r)$ where $\mu_{e}$ in the mean molecular weight per electron, $\rho(r)$ the density of matter and $N_{o}$ the Avogadro's number.

\section{NEUTRINO PRODUCTION IN THE SUN'S CORE}

The neutrino fluxes produced in the various nuclear reactions of the $\mathrm{PP}$ chains and $\mathrm{CNO}$ cycle have been computed for an updated version of the solar standard model (Turck-Chièze et al. 2004; Bahcall et al. 2005a; Guzik \& Mussack 2010; Turck-Chièze et al.|2010; Turck-Chièze \& Couvidat 2011). Figure 1 shows the location of the different neutrino emission regions of the nuclear reactions. In the Sun's core, the neutrino emission regions occur in a sequence of shells, following closely the location of nuclear reactions, orderly arranged in a sequence dependent on their temperature.

The first reaction of the $\mathrm{PP}$ chains, the $p p$ nuclear reaction, has the largest neutrino emission shell. This region extends from the center of the Sun up to $30 \%$ of the solar radius. The pep reaction has a neutrino emission shell identical to the $p p$ reaction, although only up to 25 $\%$ of the solar radius. These two key nuclear reactions are strongly dependent on the total luminosity of the star. This is the reason why different solar models with the same total luminosity produced the same $p p$ and $p e p$ neutrino emission shells. The neutrino emission shells of ${ }^{8} B-\nu$ and ${ }^{7} B e-\nu$ extend up to $15 \%$ and $22 \%$ of the solar radius. Finally, it is worth noticing that the maximum emission of neutrinos for the PP chains nuclear reactions, follows an ordered sequence (see figure 1): ${ }^{8} B-\nu,{ }^{7} B e-\nu$,

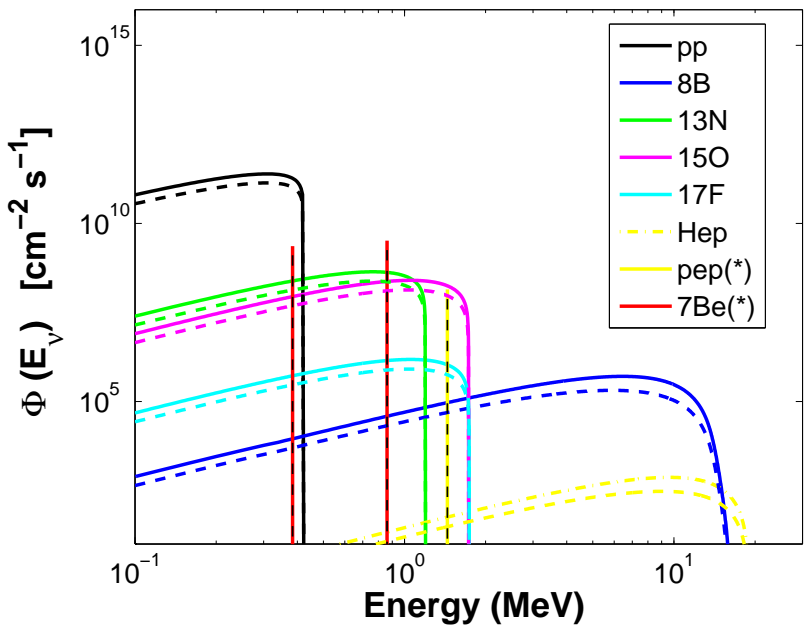

FIG. 2.- The solar neutrino energy spectrum predicted by the standard solar model. The solid curves correspond to the total neutrino fluxes produced in the various nuclear reactions of the PP chain and CNO cycle. The dashed curves correspond to electron-neutrino fluxes of the various nuclear reactions after neutrino flavour conversion. The neutrino fluxes from continuum nuclear sources are given in units of $\mathrm{cm}^{-2} \mathrm{~s}^{-1} \mathrm{Mev}^{-1}$. The line fluxes (indicated in the legend with $(*)$ ) are given in $\mathrm{cm}^{-2} \mathrm{~s}^{-1}$.

$p e p-\nu$ and $p p-\nu$ with the maximum emission located at 5 $\%, 6 \%, 8 \%$ and $10 \%$ of the solar radius, respectively.

The CNO cycle nuclear reactions produce the following electron-neutrinos sub-species: ${ }^{15} \mathrm{O}-\nu,{ }^{17} \mathrm{~F}-\nu$ and ${ }^{13} N-\nu$ released in emission shells identical to the ${ }^{8} B-\nu$. The ${ }^{13} N-\nu$ have a second emission shell located between $12 \%$ and $25 \%$ of the Sun's radius. The emission of neutrinos for ${ }^{15} O-\nu,{ }^{17} \mathrm{~F}-\nu$ and ${ }^{13} N-\nu$ is maximal at $4 \%-5 \%$ of the solar radius. The ${ }^{13} N-\nu$ neutrinos have a second emission maximum which is located at $16 \%$ of the solar radius.

\section{NEUTRINO FLAVOUR OSCILLATION IN THE SUN}

The neutrino emission reactions of the PP chains and the CNO cycle are produced at high temperatures in distinct layers in the Sun's core. Similarly, the neutrino flavour oscillations occur in the same regions. The average survival probability of electron-neutrinos in each nuclear reaction region is given by

$$
\left\langle P_{\nu_{e}}(E)\right\rangle_{j}=A_{j}^{-1} \int P_{\nu_{e}}(E, r) \Phi_{j}(r) 4 \pi \rho(r) r^{2} d r
$$

where $A_{j}$ is a normalization constant given by $A_{j}=$ $\int \Phi_{j}(r) 4 \pi \rho(r) r^{2} d r$ and $\Phi_{j}(r)$ is the electron-neutrino emission function for the $j$ nuclear reaction. $j$ corresponds to the following electron-neutrino reaction subspecies: $p p$, pep $,{ }^{8} \mathrm{~B},{ }^{7} \mathrm{Be},{ }^{13} \mathrm{~N},{ }^{15} \mathrm{O}$ and ${ }^{17} \mathrm{~F}$.

Figure 2 shows the energetic neutrino spectra (Bahcall 1989) for an updated version of our standard solar model before and after the flavour conversion. Figure 3 a shows the survival probability of electron-neutrinos produced in the regions where occur the different nuclear reactions. This survival probability of electron-neutrinos $\left\langle P_{\nu_{e}}(E)\right\rangle_{j}$ is very similar for low- and high-energy neutrinos but presents some differences between. Unfortunately, as is well known, the emitted neutrino energy spectrum is limited to a specific energy range for each nuclear reaction. Nevertheless, to highlight the sensitivity of neutrino to MSW flavour oscillations, we choose to represent the sur- 

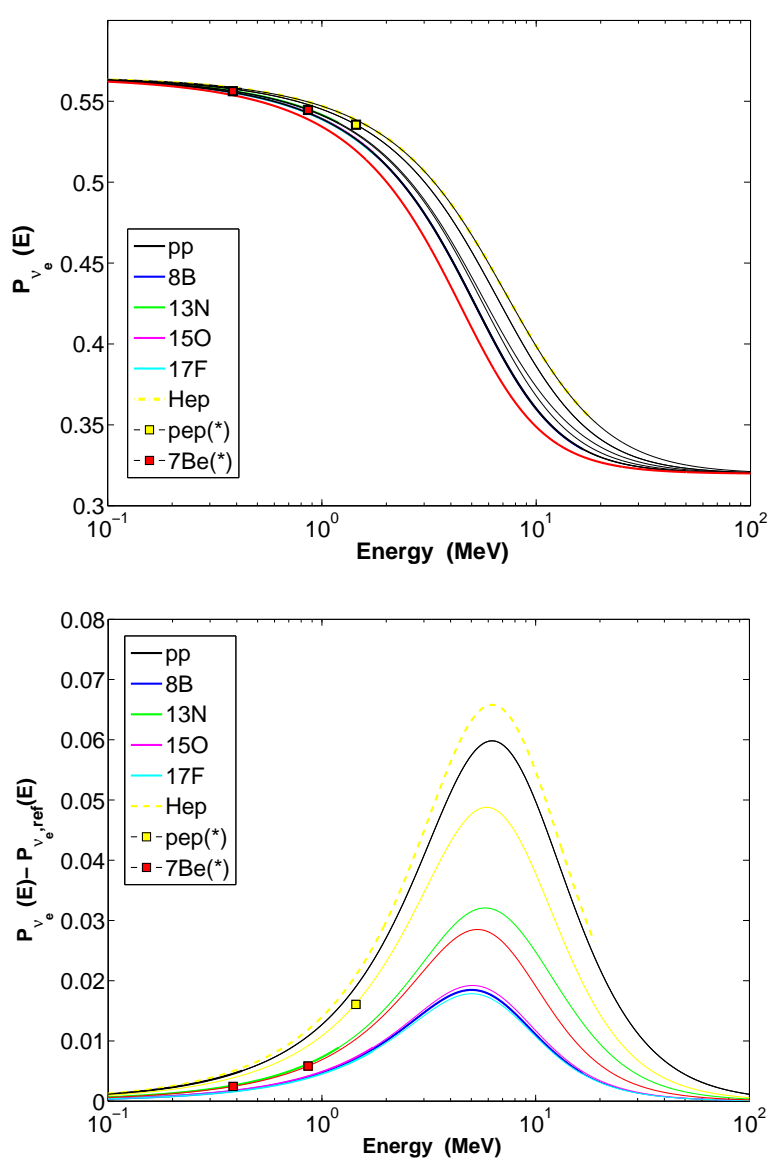

FIG. 3.- The survival probability of electron-neutrinos in function of the neutrino energy for the different regions of emission. a) survival probability of electron-neutrinos produced in the different regions b) difference between the survival probability of the different electron-neutrinos and the reference curve corresponding the central emission. The coloured part of the curves (a), and similarly the bold part of the curves (b), indicate the energy range of neutrinos produced in the Sun's core for each nuclear reaction (Cf. Figure 2). This reference curve defines the survival probability of electron-neutrinos in the centre of the Sun for which the MSW flavour oscillation mechanism is maximum (dashed red curve of figure $3 a)$.

vival probability of electron-neutrinos in all the available energy range, so that the regions of interest are indicated in colour, or by a single colour square in the case of line fluxes (Cf. Figure 2).

The neutrino change of flavour for low-energy neutrinos is due to vacuum oscillations, and for high-energy neutrinos it is caused by a cumulative effect of oscillations in vacuum and matter (MSW effect). The experimental neutrino flux measurements for low-energy neutrinos have been used to determine the value of $\theta_{12}$ (Bellini et al. 2010). The survival probability of electron-neutrinos $\left\langle P_{\nu_{e}}(E)\right\rangle_{j}$ with intermediate values of energy, between $1 \mathrm{MeV}$ and $10 \mathrm{MeV}$, have a strong dependence on the electron density of the plasma. Since the production of electron-neutrinos occurs in various nuclear reactions at different layers in the Sun's interior, a sharp differentiation is observed between the different $\left\langle P_{\nu_{e}}(E)\right\rangle_{j}$ curves for the neutrinos with intermediate values of energy. Figure 3 shows a well-ordered sequence of curves that corresponds to the difference between the survival probability of electron-neutrinos produced by each nuclear reaction $j$ and the survival probability of electron-neutrinos in the center of the Sun. This sequence of curves occurs as a result of the regular decrease of matter density from the center of the Sun. As a consequence the difference between the survival probability of electron-neutrinos produced by each nuclear reaction $j$ and the survival probability of electron-neutrinos produced in the center of the star increases with the distance of the location of the nuclear reactions to the center (Cf. Figure $3 \mathrm{~b}$ ). For example, in the case of $p p$ electron-neutrinos this difference is of the order of 0.06 which is three times larger than in the case of ${ }^{8} B$ electron-neutrinos. This is due to the fact that the ${ }^{8} B$ electron-neutrino source is located near the center of the star (Cf. Figure 1). Nevertheless, the possibility to observe such effect is somewhat limited, once the neutrino flavour oscillations induced by matter depends on the location of the neutrino source in the Sun's core (cf. Figure 1), as well as the energy of the emitted electron-neutrinos, i.e., the neutrino emission spectrum (cf. Figure 2). In the case of the $p p$ nuclear reaction, the emitted neutrinos have a maximum energy of 0.42 $\mathrm{MeV}$, consequently the survival probability difference is smaller than 0.005 . This effect is more expressive in the case of the ${ }^{8} B$ nuclear reaction for which the emitted neutrinos have a maximum energy of $14.02 \mathrm{MeV}$. In this case, the effect is maximum for neutrinos with a energy of $4 \mathrm{MeV}$ which have a survival probability difference of 0.02. Identical behaviour will be observed for the CNO nuclear reactions (namely the nuclear reactions related with the production of ${ }^{13} \mathrm{~N},{ }^{15} \mathrm{O}$ and ${ }^{17} \mathrm{~F}$ chemical elements) which emitted neutrinos with maximum energies slightly above $1 \mathrm{MeV}$, for which the survival probability difference is of the order of 0.014 . An equally pronounced difference is observed for the spectral line of pep neutrinos. In the case of the ${ }^{7} \mathrm{Be}$ neutrino the effect is important for the high-energy neutrino line and very small in the case of the small-energy neutrino line. In particular, it should be possible to check experimentally the modification of the ${ }^{8} B$ energy neutrino spectrum caused by matter flavour oscillations due to the radial dependence of ${ }^{8} B$ electron-neutrino source.

\section{THE PHYSICS OF THE SUN'S CORE AS PROBED BY NEUTRINOS}

The solar model has been checked in most of the solar radiative region by means of the high precision data of SOHO helioseismic instruments (Turck-Chièze \& Couvidat 2011; Turck-Chièze \& Lopes 2012).

As shown in equations 1 to 3 , the radial profile of the electron density of the solar standard model is a fundamental ingredient to test the neutrino physics theory. Up to now this quantity has been checked by the detection of the first gravity modes which are really sensitive to the central region of the Sun (Turck-Chièze et al. 2012; Turck-Chièze \& Lopes 2012). In the next few years, with the increase of accuracy on the measurements of several solar neutrino experiments, the situation could reverse: neutrino fluxes should start to be used to diagnose and to infer the thermodynamic properties of the Sun's core.

The neutrino flux emissions inside the Sun are sensitive to the local values of temperature (specifically some of them), matter density, chemical composition and electronic density where the nuclear reactions are taking 
Central PARAmeters of STANDARD AND MODIFIED SOLAR MODELS

\begin{tabular}{lccc}
\hline Solar Models & SSM & Sun A & Sun B \\
\hline \hline $\begin{array}{l}\text { Model Values } \\
\text { density }\left(\mathrm{g} / \mathrm{cm}^{3}\right) \\
\text { mean molecular } \\
\text { weight per electron } \mu_{e}\end{array}$ & 151 & 161 & 167 \\
\hline $\begin{array}{l}\text { Neutrino Energy (MeV) } \\
\text { Lower Cut-off E } \mathrm{rc}^{\mathrm{b}}\end{array}$ & 2.0 & 1.65 & 1.64 \\
\hline \hline $\begin{array}{l}\text { Inverted Values } \\
\text { density }\left(\mathrm{g} / \mathrm{cm}^{3}\right)\end{array}$ & 1.83 & 1.75 \\
$\begin{array}{l}\text { with } \mu_{e} \text { solar model } \\
\text { density }\left(\mathrm{g} / \mathrm{cm}^{3}\right)\end{array}$ & $151.5[0.3 \%]$ & $161[+0.4 \%]$ & $168[+0.6 \%]$ \\
with $\mu_{e}=\mu_{\mathrm{SSM}}=1.69$ & $151.5[0.3 \%]$ & $165[+2.8 \%]$ & $173[+3.6 \%]$ \\
\hline \hline
\end{tabular}

The solar standard model (SSM) and the modified models, Sun A and Sun B, were calibrated in order to keep the total luminosity of the present Sun ( Figure 4 shows the corresponding density profile).

b $E_{r c}$ is the minimum energy that a neutrino must have to be affected by the MSW flavour oscillation mechanism. The values between $[\ldots]$ show the percentage difference between the model values and the inverted values.

place.

In particular, as the MSW flavour oscillation is strongly dependent on the local electronic density, it is possible to infer this quantity from the survival probability of electron-neutrinos. Furthermore, since electronic density depends on matter density and chemical composition, it will also be possible to obtain some information about these quantities.

\subsection{The sensitivity of neutrino flavour oscillations to the central density}

Any standard solar model calibrated for the present day total solar luminosity has a radial profile of temperature and density in the core that results from the balance found between the energy produced by the nuclear reactions and the energy transported to the surface. Solar models with slightly different physical assumptions, have different radial profiles of temperature and matter density among other quantities. This is the result of the readjustment of the Sun's internal structure caused by the need to obtain the same total luminosity.

Our evolution code is an up-to-date version of the one-dimensional stellar evolution code CESAM (Morel 1997). The code has an up-to-date and very refined microscopic physics (updated equation of state, opacities, nuclear reactions rates, and an accurate treatment of microscopic diffusion of heavy elements), including the solar mixture of Asplund et al. (2005, 2009). The solar models are calibrated to the present solar radius $R_{\odot}=6.9599 \times 10^{10} \mathrm{~cm}$, luminosity $L_{\odot}=3.846 \times$ $10^{33} \mathrm{erg} \mathrm{s}^{-1}$, mass $M_{\odot}=1.989 \times 10^{33} \mathrm{~g}$, and age $t_{\odot}=4.54 \pm 0.04$ Gyr (e.g. Turck-Chièze \& Couvidat 2011; Turck-Chièze, Piau \& Couvidat 2011b). The models are required to have a fixed value of the photospheric ratio $(Z / X)_{\odot}$, where $\mathrm{X}$ and $\mathrm{Z}$ are the mass fraction of hydrogen and the mass fraction of elements heavier than helium. The value of $(Z / X) \odot$ is determined according to the solar mixture proposed by Asplund et al. (2005). Our reference model is a solar standard model that shows acoustic seismic diagnostics and solar neutrino fluxes near from other solar standard models (Turck-Chièze \& Lopes 1993; Turck-Chièze et al. 2004; Bahcall et al. 2005b; Guzik \& Mussack 2010; Serenelli et al. 2009; Turck-Chièze et al. 2010).

In Table 1 we present the central density and the mean electronic molecular weight of this standard solar model and of two other non-standard solar models. These last two models have their radiative energy transport slightly modified in the core in order to obtain a Sun's model with a higher central density. Figure 4 a shows the radial density profile in the Sun's core for the three models. The amount of neutrinos converted to the nonelectron flavour by the MSW flavour oscillation mechanism is dependent on the central density profile. Figure $4 \mathrm{~b}$ shows the difference between the survival probability of electron-neutrinos for each of the two modified solar models relatively to the standard solar model. The difference is more significant for the model with the highest central density. It is clearly illustrated that an increase of the central density leads to an increase in the neutrinos converted by the MSW flavour oscillation in all electron-neutrino subspecies.

The survival probability of electron-neutrinos can be used to infer the matter density in the centre of the Sun, once that precise neutrino measurements will become available. In the following we describe a simple procedure that highlights the sensitivity of neutrino flavour oscillations to the Sun's core density. Only neutrinos with energy above a given minimum value will have their flavour changed by the MSW flavour oscillation mechanism. Several resonances occur in equation (2) for neutrinos with energy $E_{r}$ such that $V\left(E_{r}, r\right)=\Delta m^{2} \cos \left(2 \theta_{v}\right)$. For the lowest neutrino energy value $E_{\mathrm{r}}$ that verifies the previous equation, we choose to call it $E_{\mathrm{rc}^{-}}$the cut-off minimum resonance neutrino energy for a given solar model. The value of $E_{\mathrm{r}}$ is minimum for the maximum value of the density of matter (equation 3). Therefore, each solar model has a unique cut-off minimum neutrino energy $E_{\mathrm{rc}}$ given by

$$
E_{\mathrm{rc}}=\frac{\Delta m^{2} \cos \left(2 \theta_{v}\right)}{2 \sqrt{2} G_{f} N_{o} \rho_{c} / \mu_{e c}}
$$

where $\rho_{c}$ in the central matter density and $\mu_{e c}$ is the central value of mean molecular weight per electron.

In Table 1, we show the value of $E_{\mathrm{rc}}$ for the three solar models computed from the survival probability electronneutrino function: if the resonance condition is verified, $\theta_{m}=\pi / 4$ and the survival probability of electronneutrinos (equation 1) has the specific value of 0.5 . The value $E_{r c}$ is determined for each solar model by identifying the neutrino minimum energy value for which the survival probability of electron-neutrino is equal to 0.5 . The models with the higher central densities have the lowest values of $E_{\mathrm{rc}}$ (see Table 1 ). The central density can be calculated from equation (15) once the value of $E_{\mathrm{rc}}$ is known for each solar model and assuming that $\mu_{e c}$ (or the chemical composition) is known. In Table 1 we show the "inferred values" of the central density. The small variation $\mu_{e c}$ between different models allows us to make a reasonable estimation of the central density, assuming that the variation of resonance energy $E_{r c}$ is due solely to variations in the central density. The central density is 

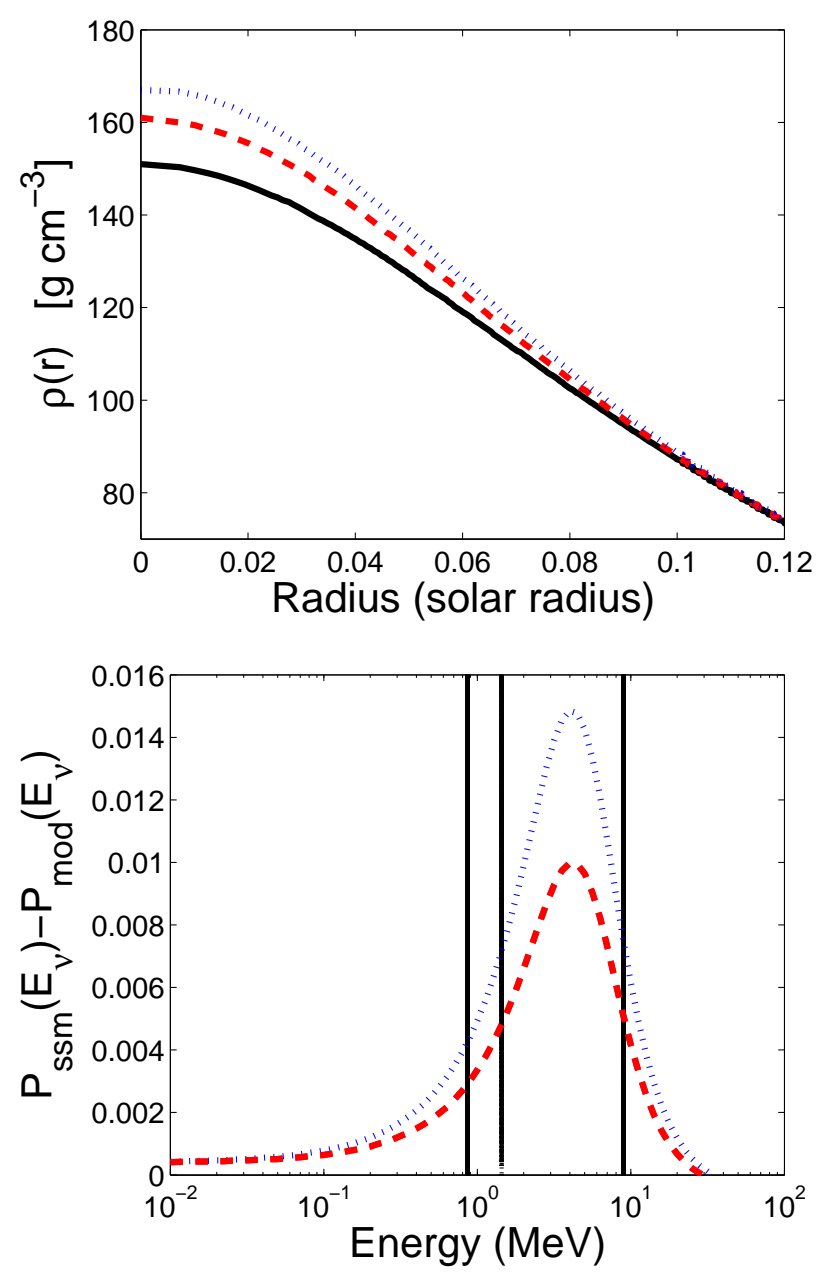

FIG. 4. - The standard solar model and two modified solar models (see table 1): (a) density as a function of radius in the solar core; (b) difference of the survival probability of electron-neutrinos of two modified solar models and the survival probability of electronneutrinos of the standard solar model. The survival probability of electron-neutrinos was computed for neutrinos propagating in the center of the star. The continuous-black curve corresponds to the standard solar model, the dashed-red curve and dotted-blue curve correspond to the solar models, Sun A and Sun B (see table 1). In the figure, the vertical lines indicate the location of the present solar neutrino measurements: ${ }^{7} \mathrm{Be}-\nu(0.56 \mathrm{MeV})$, pep- $\nu(1.44 \mathrm{MeV})$ and ${ }^{8} B-\nu(8.9 \mathrm{MeV})$.

inverted at most with a difference of a few percent higher than the value of the model, even in the case that $\mu_{e c}$ is poorly known.

In fact, the central value of the density in the Sun might be more difficult to obtain since the electronneutrinos are not produced just in the center, and the different ranges of energy of neutrinos are limited (Cf. Figure 2). An interesting possibility is to probe the solar core by using the neutrinos produced near the centre of the star, such as ${ }^{8} B-\nu,{ }^{15} O-\nu$ and ${ }^{17} F-\nu$ neutrino fluxes. The simultaneous measurement of two different neutrino fluxes produced in the same layer of the Sun's interior, such as ${ }^{8} B-\nu$ and ${ }^{15} O-\nu$, could be used to estimate simultaneously the matter density and the average molecular weight per electron in the core of the Sun.

\subsection{The sensitivity of neutrino flavour oscillations to the radial density profile}

All the solar models validated by acoustic and gravity mode detections, predict that the density of matter decreases by $90 \%$ from its central value to the value at $30 \% \mathrm{R} \odot$. The neutrino fluxes produced by the different nuclear reactions are sensitive to the local values of the electronic density. The different neutrino fluxes $\Phi_{j}(r)$ can be interpreted as a local average of $n_{j}(r)$.

In principle taking into account the high sensitivity of solar neutrino fluxes to the temperature changes in the solar core, we should expect important variations in the location of the neutrino source in the Sun's core. However, the effect is actually quite small. The reason is related with the calibration procedure that solar models are subjected to, which require that the Sun in the present age must have the observed solar luminosity. Consequently, the production of neutrinos inside the Sun, which are strongly dependent on the luminosity of the star, namely, the neutrinos produced in the proton-proton chain nuclear reactions, such as ${ }^{8} B$ and ${ }^{7} B e$, occur sensibly in the same location, i.e., at the same distance from the center of the star. As an example, solar models which experiment a decrease of $10 \%$ in the central density (and a $3 \%$ reduction in the central temperature) have a variation of the maximum of any of the neutrino sources (both proton-proton chain nuclear reactions and $\mathrm{CNO}$ nuclear reactions) displaced by an amount smaller than $1 \%$ of the solar radius, although the neutrino fluxes change significantly due to their high sensitivity to the temperature. Actually, the proton-proton neutrino source has the same location, as this nuclear reaction, more than any other depends directly from the total luminosity of the star. For the purpose of this article the neutrino sources of different solar models are considered to be located within the same fraction of the solar radius. Therefore, if the fundamental parameters of neutrino oscillations are known, the probability of survival of electron neutrinos can be used to measure the radial electronic density profile in the solar core, i.e., the matter density and molecular weight per electron in several layers of the Sun. A simple procedure to obtain the electronic density is now proposed. We consider first an electronic-neutrino survival probability $\bar{p}_{j}$ determined, at a specific neutrino energy $E_{j}$, by some neutrino experiment. One assumes that $\bar{p}_{j}$ is determined fully independently from the solar standard model by a method identical to the one described in Berezinsky \& Lissia (2001). Preferentially, $E_{j}$ is the value chosen within the energy interval for which the MSW neutrino flavour oscillations are significant (cf. Figure 3). For each pair $\left(E_{j}, \bar{p}_{j}\right)$, the value of the electronic density for a certain layer can be computed from equations (13). We estimate $\bar{n}_{j} \equiv\left\langle n_{e}(r)\right\rangle_{j}$ corresponding to electronic density of several layers in the Sun's core, as $\bar{n}_{j}=1 /\left(2 \sqrt{2} G_{f}\right) \times \alpha_{j}\langle V\rangle_{j} / E_{j}$, where $\langle V\rangle_{j}$ is computed from $p_{j}$ using the previous equations, and $\alpha_{j}$ is a weight-correction parameter unique for each $j$ nuclear reaction. $\alpha_{j}$ is estimated for the solar standard model and it takes a value of the order of a unit, increasing slightly when the neutrino source $\Phi_{j}(r)$ moves

$6 \mathrm{n}_{\mathrm{j}}(\mathrm{r})$ is the estimated mean value of the electronic density for the layer at the radius $\mathrm{r}_{\mathrm{j}}$, where the neutrino source $\Phi_{j}(r)$ is maximum (Cf. figure 1). 
away from the centre of the star. Similarly, by using the experimental value $\bar{p}_{j}$, which is different from the theoretical prediction $p_{j}$, we estimate the value $\bar{n}_{j}$ as a correction to the theoretical value $n_{j}$, from the equation $\Delta n_{j} / n_{j}=\beta_{j} \Delta p_{j} / p_{j}$, where $\Delta n_{j}=\bar{n}_{j}-n_{j}$, $\Delta p_{j}=\bar{p}_{j}-p_{j}$ and $\beta_{j}$ is a coefficient computed from the solar standard model. The previous expression is obtained from a perturbation analysis of equations (1, 3). In this analysis $\bar{n}_{j}$ is the inverted value deduced from the experimental data.

Figure 5 shows the inverted electronic density values obtained from the survival probability of electronneutrinos as described previously. There is a good agreement between the values of the electronic density obtained by inversion and the electronic density of the standard solar model. On Figure 5 a we show the electronic density values that one can deduce from the survival probabilities obtained from the neutrino detector measurements. More specifically, we present the electronic density inverted from the ${ }^{7} \mathrm{Be}$ neutrino fluxes using the measurements of Bellini et al. (2010) and the ${ }^{8} B$ neutrino fluxes using the measurements of Aharmim et al. (2007).

The Borexino experiment measures ${ }^{7} \mathrm{Be}$ solar neutrino rates with an accuracy better than $5 \%$. This corresponds to a ${ }^{7} \mathrm{Be}$ neutrino flux $\Phi\left({ }^{7} \mathrm{Be}\right)=4.87_{-0.24}^{+0.24} \times$ $10^{9} \mathrm{~cm}^{-2} \mathrm{~s}^{-1}$, under the assumption of the MSWLMA scenario of solar neutrino oscillations Bellini et al. 2011a; Arpesella et al. 2008). The estimated survival probability, assuming a high metallicity solar standard model, was initially estimated to be $P_{\nu_{e}}\left({ }^{7} \mathrm{Be}\right)=0.56 \pm 0.1$ at the energy $0.862 \mathrm{MeV}$ (Arpesella et al. 2008). Latter the Borexino collaboration(Bellini et al. 2011a) updated their estimation to $P_{\nu_{e}}\left({ }^{7} B e\right)=0.52_{-0.06}^{+0.07}$. Similarly, as proposed by Berezinsky \& Lissia (2001), we compute the survival probability for ${ }^{8} B$ as $P_{\nu_{e}}\left({ }^{8} B\right)=$ $\Phi_{\nu_{e}}\left({ }^{8} B\right) / \Phi_{t}\left({ }^{8} B\right)$, where $\Phi_{t}\left({ }^{8} B\right)$ is the total neutrino flux integrating all neutrino flavours measured by SNO (Aharmim et al. 2010): $\Phi_{t}\left({ }^{8} B\right)=5.046_{-0.275}^{+0.226} \times$ $10^{6} \mathrm{~cm}^{-2} \mathrm{~s}^{-1}$ and $\Phi_{\nu_{e}}\left({ }^{8} B\right)$ is the electron-neutrino flux measured by the same detector (Aharmim et al. 2007): $\Phi_{\nu_{e}}\left({ }^{8} B\right)=1.76_{-0.14}^{+0.14} \times 10^{6} \mathrm{~cm}^{-2} \mathrm{~s}^{-1}$. It follows that $P_{\nu_{e}}\left({ }^{8} B\right)=0.35 \pm 0.047$ for SNO. It is clear that presently the error bars are still too large to really estimate the small gap with the standard model values. But one may hope a progress on the future as we have already for ${ }^{8} B$ neutrinos several improved detections: the neutrino flux measured by the Kamiokande-III experiment (Abe et al. 2011),$\Phi_{\nu_{e}}\left({ }^{8} B\right)=2.32_{-0.09}^{+0.09} \times 10^{6} \mathrm{~cm}^{-2} \mathrm{~s}^{-1}$, the neutrino flux measured by the Borexino experiment is $\Phi_{\nu_{e}}\left({ }^{8} B\right)=2.4_{-0.5}^{+0.5} \times 10^{6} \mathrm{~cm}^{-2} \mathrm{~s}^{-1}$ (Bellini et al. 2010) Moreover, we already measure the energy dependence of these fluxes down to $3 \mathrm{MeV}$. So, to illustrate the potential of this diagnostic and to indicate some research perspectives, we present in Figure 5b the electronic density values deduced assuming an error bar on the survival probability of the order of $4 \%$. It follows that the error bar on the electronic density that one can deduce for ${ }^{7} \mathrm{Be}$ and ${ }^{8} \mathrm{~B}$ fluxes is largely reduced. This is due to the high sensitivity of neutrino flavour oscillations to the electronic density. This first study shows the potentiality of such type of neutrino diagnostic. Another possibility
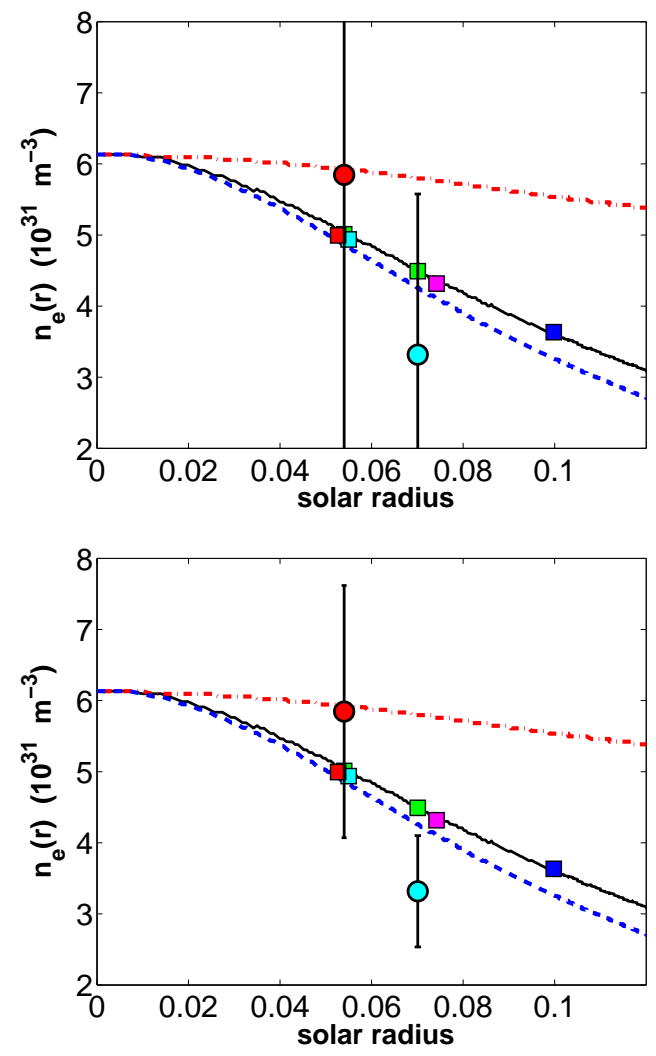

FIG. 5.- Radial profile of the electronic density $n_{e}(r)$ (black continuous curve) in the core of the Sun of the solar standard model, on the same plot appear normalized values of the radial profile of the density of matter, central $\rho=167 \mathrm{~g} \mathrm{~cm}^{-3}$ (blue dashed curve) and the radial profile of molecular weight per electron $\mu_{e}(r)$, central value of 1.69 (red dotted curve). The points shown in the figure correspond to the values of density inverted from the survival probability of electron-neutrinos, at the following energies: $0.56 \mathrm{MeV}\left({ }^{7} \mathrm{Be}\right), 1.44 \mathrm{MeV}(p e p), 8.9 \mathrm{MeV}\left({ }^{8} B\right), 1.0 \mathrm{MeV}\left({ }^{13} N\right)$, $1.0 \mathrm{MeV}\left({ }^{15} \mathrm{O}\right) 1.0 \mathrm{MeV}\left({ }^{17} F\right)$. The location of the source are the following ones: $0.070 R_{\odot}\left({ }^{7} \mathrm{Be}\right.$, square-green), $0.099 R_{\odot}(\mathrm{PeP}$, square-blue), $0.054 R_{\odot}\left({ }^{8} B\right.$, square-green $), 0.074 R_{\odot}\left({ }^{13} N\right.$, squaremagenta), $0.055 R_{\odot}\left({ }^{15} \mathrm{O}\right.$, square-cyan $), 0.053 R_{\odot}\left({ }^{17} \mathrm{~F}\right.$, squarered). The inverted electronic density computed from measured ${ }^{8} B$ and ${ }^{7} \mathrm{Be}$ neutrino fluxes: (a) the values computed from the survival neutrino probability obtained from the present results of SNO $\left({ }^{8} B\right)$ and Borexino $\left({ }^{7} \mathrm{Be}\right)$, (b) idem considering that the experimental error in the determination of the survival probability of electron neutrinos is $4 \%$. The red and in cyan circles correspond to the ${ }^{8} B$ and ${ }^{7} \mathrm{Be}$ neutrino measurements respectively.

is to fix values of the electron-neutrino survival probability curve for neutrinos of low and high energy, as such neutrinos have respectively pure vacuum oscillations and vacuum plus MSW oscillations. In this case neutrinos with energy in the interval $0.5-10 \mathrm{MeV}$, can probe the electronic density of different solar layers.

\section{DISCUSSION AND CONCLUSION}

The capability to make such a study is real because new neutrino detection experiments are arriving and others are planned for the near future. The diversity of neutrino flux measurements expected at different energy levels will allow us to make reliable and detailed neutrino inversions.

In this first study we have used only two mean survival probabilities, without taking into account the energy dependence of the boron flux and the detailed radial 
distribution. But the Borexino detector also measures the $p e p$ neutrino flux, $\Phi($ pep $)=1.6_{+0.3}^{+0.3} \times 10^{8} \mathrm{~cm}^{-2} \mathrm{~s}^{-1}$ (Bellini et al. 2012). This preliminary result is still insufficiently accurate, but it is not in contradiction with the fact that the Sun is hotter than what is suggested by the present standard solar model. The pep neutrino flux is strongly dependent on the luminosity of the star. Therefore, it is an indirect measurement of the total energy (luminosity) produced in the nuclear region. So it represents a powerful probe to the physics of the nuclear region of the Sun, in parallel to the information introduced in the seismic model (Turck-Chièze \& Lopes 2012).

Today, there is some uncertainty about the chemical composition in the Sun's core. In parallel, all the neutrino measurements agree with the predicted values of the solar seismic model but not so well with the standard model (Turck-Chièze \& Couvidat 2011), showing some capability of understanding the difference with the standard model if one extracts the electronic density profile. The results obtained so far suggest that in the near future we will be able to probe the physics of the Sun's core using neutrinos.

In this work we have proposed a strategy that allows to use the solar neutrino flux measurements to invert the electron density of the solar plasma at specific locations of the Sun's core. Furthermore, in the near future, it will also be possible to put important constraints on the matter density and on the molecular weight per electron of the solar plasma. The inversion is made based upon the assumption that the future Earth neutrino experiments (e.g., reactor experiments, superbeams, beta beams and neutrino factories) will allow the precise determination of basic neutrino oscillation parameters.

Today, the neutrino fluxes of ${ }^{7} B e-\nu,{ }^{8} B-\nu$ and $p p-\nu$ are already well measured and obtained separately. The $p e p-\nu$ neutrino flux is also measured although with much less precision. It will be interesting to verify if such results hold with the increase of accuracy in the observations within the next few years. The present electronicneutrino survival probability curve is fixed at low neutrino energy by $p p-\nu$, sensitive to pure vacuum oscilla- tions, and by ${ }^{8} B-\nu$, sensitive to vacuum plus MSW oscillations. Therefore it will be relevant to verify if future measurements of ${ }^{7} B e-\nu$ and $p e p-\nu$ can give us some information about the electronic density in two distinct layers of the Sun, namely $0.1 R_{\odot}(p e p-\nu)$ and $0.07 R_{\odot}\left({ }^{7} B e-\nu\right)$. There is a real possibility that Borexino or SNO + experiments will be able to measure accurately these different neutrino fluxes, as well as the neutrino fluxes of some of the CNO cycle neutrino emission reactions.

The LENA (Low Energy Neutrino Astrophysics) solar neutrino detector is expected to start to operate within the next few years. This detector will be able to perform very accurate measurements of the different sources of the solar neutrino spectrum, allowing not only very precise measurements of the solar core plasma, but also to identify possible seasonal neutrino variations.

In conclusion, we have shown that in the near future one may hope to use neutrino spectroscopic measurements to infer the electronic density of the plasma in the core of the Sun. This will be an important and totally independent test of the nuclear region of the Sun properties in parallel with the helioseismic inversions of matter density and sound speed. The proposed method, that could be improved, allows to infer the properties of the plasma in the very central region of the Sun, which until now have only been explored by some gravity mode detections with GOLF/SoHO. We have shown in table 1 that the inversion of different density profile can be done with a reasonable quality if the neutrino fluxes are obtained with a good accuracy. Of course this method supposes that the neutrino oscillations description is independent of solar model predictions. If that becomes the case, one will be able to extract from the different solar neutrino detections, the electronic density and matter density profiles with some hints on the CNO composition in the core of the Sun.

This work was supported by grants from "Fundação para a Ciência e Tecnologia" and "Fundação Calouste Gulbenkian". We thank the anonymous referee for the helpful comments that much improved the clarity of the paper.

\section{REFERENCES}

Abe, K., et al. 2011, Phys. Rev. D, 83, 52010

Aharmim, B., et al. 2007, Phys. Rev. C, 75, 45502

Aharmim, B., et al. 2010, Phys. Rev. C, 81, 55504

Ahmad, Q. R., et al. 2001, Phys. Rev. Lett., 87, 71301

Apollonio, M. \& Baldini, A. 1999, Physics Letters B, 466, 415

Arpesella, C., et al. 2008, Phys. Rev. Lett., 101, 91302

Asplund, M., Grevesse, N., \& Sauval, A. J. 2005, Cosmic

Abundances as Records of Stellar Evolution and

Nucleosynthesis in honor of David L. Lambert, 336, 25

Asplund, M., Grevesse, N., Sauval, A. J., \& Scott, P. 2009,

Annual Review of Astronomy and Astrophysics, 47, 481

Bahcall, J. N. 1989, Cambridge and New York, Cambridge University Press, 1989, 584 p., -1

Bahcall, J., Basu, S., Pinsonneault, M., \& Serenelli, A. M. 2005a, ApJ, 618, 1049

Bahcall, J. N., Serenelli, A. M., \& Basu, S. 2005b, ApJ, 621, L85

Balantekin, A. \& Yuksel, H. 2003, Journal of Physics G: Nuclear and Particle Physics, 29, 665

Bellini, G., et al. 2012, Phys. Rev. Lett., 108, 51302

Bellini, G., et al. 2011a, Phys. Rev. Lett., 107, 141302

Bellini, G., et al. 2010, Phys. Rev. D, 82, 33006

Bellini, G., et al. 2011b, Physics Letters B, 696, 191

Berezinsky, V. \& Lissia, M. 2001, Physics Letters B, 521, 287
Bilenky, S. 2010, 'Introduction to the Physics of Massive and Mixed Neutrinos', Lect. Notes Phys. 817 (Springer, Berlin Heidelberg 2010), DOI 10.1007/978-3-642-14043-3.

Davis, R. 1964, Phys. Rev. Lett., 12, 303

Eguchi, K., et al. 2003, Phys. Rev. Lett., 90, 21802

Fogli, G. L., Lisi, E., Marrone, A., Palazzo, A., \& Rotunno, A. M. 2009, eprint arXiv:0905.3549

Fogli, G. L.,Lisi, E., Marrone, A., Montanino, D., Palazzo, A., Rotunno, A. M. 2012, Phys. Rev. D, 86, 3012

Fukuda, S., et al. 2001, Phys. Rev. Lett., 86, 5651

Gando, A., et al. 2011, Phys. Rev. D, 83, 52002

Gonzalez-Garcia, M. C. \& Maltoni, M. 2008, Physics Reports, 460,1

Gonzalez-Garcia, M. C. et al. 2012, arXiv:1209.3023

Guzik, J. A. \& Mussack, K. 2010, ApJ, 713, 1108

Hernandez, P. 2010, arXiv, 1010, 4131

Landau, L. \& Rosenkewitsch, L. 1932, Zeitschrift fur Physik, 78, 847

Lisi, E. \& Montanino, D. 1997, Physical Review D (Particles and Fields), 56, 1792

Morel, P. 1997, A \& A Supplement series, 124, 597

Pallavicini, M., et al. 2011, Nuclear Instruments and Methods in Physics Research Section A, 630, 210 
Schwetz, T., Tórtola, M., \& Valle, J. W. F. 2008, New J. Phys., 10,3011

Serenelli, A. M., Basu, S., Ferguson, J. W., \& Asplund, M. 2009, The Astrophysical Journal Letters, 705, L123

Turck-Chièze, S. \& Lopes, I. 1993, Astrophysical Journal, 408, 347

Turck-Chièze, S. \& Couvidat, S. 2011, Rep. Prog. Phys., 74, 6901

Turck-Chièze, S. \& Lopes, I. 2012, Res. Astron. AstroPhys., 12, number 8, 1107

Turck-Chièze, S., Palacios, A., Marques, J. P., \& Nghiem, P. A. P. 2010, ApJ, 715, 1539
Turck-Chièze, S., Piau, L., \& Couvidat, S. 2011b, The Astrophysical Journal Letters, 731, L29

Turck-Chièze S., Couvidat S., Piau L. et al. 2004, Phys. Rev. Lett., 93, 211102

Turck-Chièze, S., Garcia, R. A., Lopes, I., Ballot, J., Couvidat, S., Mathur, S., Salabert, D., \& Silk, J. 2012, The Astrophysical Journal Letters, 746, L12

Wolfenstein, L. 1978, Phys. Rev. D, 17, 2369

Zener, C. 1932, Royal Society of London Proceedings Series A, 137, 696 